\title{
Australian perspectives: outcomes from the 2015 Australia and New Zealand Developmental Origins of Health and Disease (ANZ DOHaD) scientific meeting
}

The papers in this themed issue represent work presented and discussed at the 2015 ANZ DOHaD (Australia and New Zealand Developmental Origins of Health and Disease) scientific meeting in Melbourne. The ANZ DOHaD convened working groups across several areas of $\mathrm{DOHaD}$ to identify gaps in knowledge and foster collaboration to close these gaps. Prescott et al. summarize the discussions of these working groups.

Davies et al. ${ }^{1}$ for the Early Life Nutrition Working Group report on the opportunity for improving long-term health in Australia and New Zealand through altering diet in childhood. The paper highlights that nutrition is one of the most easily modifiable environmental factors during early life with known benefits for health throughout the lifespan. The authors recommend preparation and dissemination of guidelines for optimizing early life nutrition as part of a preventative health policy agenda for Australia and New Zealand.

Led by Dickinson et al., ${ }^{2}$ the animal models for $\mathrm{DOHaD}$ Research Working Group reviewed the major principles of study design for animal models to maximize relevance, reproducibility and translation of knowledge into improving health and well-being. The review provides recommendations for consideration in species/strain choice, methods for inducing pregnancy, environment, biological replicates, offspring sex and tissue collection methods. The authors suggest a shift in the approach to $\mathrm{DOHaD}$ research; in a time of limited resources, the field will benefit from expanding collaborative networks to provide access to existing animal preparations, tissue banks and experimental data.

Prize winners at the ANZ $\mathrm{DOHaD}$ scientific meeting were invited to prepare and submit a manuscript based on their presented data. We congratulate the prize winners Jacinta Kalisch-Smith, Richard Schlegel, Clare Reynolds, Jen Ni Cheong, Amy Ashman and Chantal Pileggi.

Schlegel $e t ~ a l .^{3}$ explore the impact of maternal magnesium deficiency on cardiorenal function in offspring in mice. They report little difference between offspring of control and magnesium-deficient mothers. The authors conclude that mild magnesium deficiency in mouse pregnancy does not have an impact on long-term offspring cardiorenal health.

Ashman et al. ${ }^{4}$ provide important information about nutrition in Indigenous Australian women and their infants. The authors studied 79 mother-child dyads from the Gomeroi gaaynggal cohort. Breastfeeding was initiated by $85.9 \%$ of mothers; however, the median duration of breastfeeding was only 1.4 months (0.5-4 months). Compared with recommendations, maternal nutrient intakes of fibre, folate, iodine, calcium, potassium and vitamin $\mathrm{D}$ were low and proportions of energy from fat were high. The authors suggest that nutritional support is needed for Indigenous Australian women and their children to improve long-term health.

The working group concept developed by ANZ $\mathrm{DOHaD}$ has been productive and will continue. In time these working groups will result in the development of guidelines aimed to promote $\mathrm{DOHaD}$ and $\mathrm{DOHaD}$ research, promote collaboration and highlight excellent research in our region through prizes and the opportunity for early career researchers to publish their work in our disciplines journal.

\section{References}

1. Davies PSW, Funder J, Palmer DJ, et al. Early life nutrition and the opportunity to influence long-term health: an Australasian perspective. J Dev Orig Health Dis. 2016; 7, 440-448.

2. Dickinson H, Moss TJ, Gatford KL, et al. A review of fundamental principles for animal models of $\mathrm{DOHaD}$ research: an Australian perspective. J Dev Orig Health Dis. 2016; 7, 449-472.

3. Schlegel RN, Moritz KM, Paravicini TM. Maternal hypomagnesemia alters renal function but does not program changes in the cardiovascular physiology of adult offspring. J Dev Orig Health Dis. 2016; 7, 473-480.

4. Ashman AM, Collins CE, Weatherall LJ, et al. Dietary intakes and anthropometric measures of Indigenous Australian women and their infants in the Gomeroi gaaynggal cohort. J Dev Orig Health Dis. 2016; 7, 481-497.

H. Dickinson The Ritchie Centre, Hudson Institute of Medical Research Melbourne, VIC, Australia

J. L. Morrison

Early Origins of Adult Health Research Group, School of Pharmacy and Medical Sciences, Sansom Institute for Health Research University of South Australia, Adelaide, SA, Australia 